\title{
Bilateral Renal Infarctions During the Use of Sumatriptan
}

\section{Blaise Abramovitz ${ }^{1}$, Amanda Leonberg-Yoo ${ }^{1}$, Jehan Z. Bahrainwala ${ }^{1}$, Harold Litt $^{2}$ and} Michael R. Rudnick ${ }^{1}$

\begin{abstract}
${ }^{1}$ Renal Electrolyte and Hypertension Division, Perelman School of Medicine of the University of Pennsylvania, Philadelphia, Pennsylvania, USA; and ${ }^{2}$ Department of Radiology, Perelman School of Medicine of the University of Pennsylvania, Philadelphia, Pennsylvania, USA
\end{abstract}

Correspondence: Blaise Abramovitz, 3096 Henrich Farm Lane, Allison Park, Pennsylvania 15101, USA. E-mail: blaise. abramovitz@uphs.upenn.edu

Kidney Int Rep (2018) 3, 1233-1236; https://doi.org/10.1016/j.ekir.2018.05.003

(C) 2018 International Society of Nephrology. Published by Elsevier Inc. This is an open access article under the CC BYNC-ND license (http://creativecommons.org/licenses/by-nc-nd/4.0/).

\section{INTRODUCTION}

Trerin riptans are effective as acute abortive therapy for migraine and cluster headaches by targeting the serotonin 5-HT $\mathrm{T}_{1 \mathrm{~B} / \mathrm{1D}}$ receptors located in the cerebral, coronary, and peripheral arteries. This class of medications inhibits the release of vasoactive peptides and promotes vasoconstriction, combating the pathologic vasodilatation contributing to migraine headaches. ${ }^{1}$ Potential complications of these vasoconstrictive properties include myocardial infarction, ${ }^{2,3}$ cerebrovascular accident, ${ }^{4}$ ischemic colitis, ${ }^{5}$ and spinal cord infarction. ${ }^{6}$ We present a case of bilateral renal infarctions associated with sumatriptan use.

\section{CASE PRESENTATION}

A 45-year-old white woman with a history of migraine headaches presented to the emergency department with acute left-sided abdominal and lower back pain. She denied symptoms of dysuria or hematuria, and did not report any new medication use. Home medications included sumatriptan $25 \mathrm{mg}$ as needed for migraine headaches, which were used shortly prior to presentation, and an intrauterine device. She had no personal or family history of a hypercoagulable state. She was a former smoker but denied the use of illicit drugs, including cocaine. She was diagnosed with presumed pyelonephritis and was discharged with trimethoprim-sulfamethoxazole, a nonsteroidal anti-inflammatory drug, and a proton pump inhibitor.

One week later, she re-presented to the emergency department with persistent left-sided abdominal pain. On arrival, her temperature was $98.8^{\circ} \mathrm{F}$, blood pressure was $145 / 82 \mathrm{~mm} \mathrm{Hg}$, heart rate was $70 \mathrm{bpm}$ and regular, and her left upper quadrant and epigastrium were tender to palpation. Laboratory tests were as follows: serum creatinine $0.97 \mathrm{mg} / \mathrm{dl}$ (reference range, 0.57-1.00), blood urea nitrogen $8 \mathrm{mg} / \mathrm{dl}$ (reference range, 6-24), hemoglobin $12.1 \mathrm{~g} / \mathrm{dl}$ (reference range, 11.1-15.9), prothrombin time 11.4 seconds (reference range, 12.2-14.2), partial thromboplastin time 31 seconds (reference range, 20.8-34.4), and lactate dehydrogenase $209 \mathrm{IU} / \mathrm{l}$ (reference range, 90-190 IU/l), and urinalysis was negative for blood and leukocyte esterase. A computed tomography (CT) examination of the abdomen/pelvis with i.v. contrast showed a patchy wedge-shaped area of decreased enhancement in the lower pole of the left kidney, concerning for a renal infarct (Figure la), with thrombosis of a segmental artery to this region (Figure $1 \mathrm{~b}$ ). A transthoracic echocardiogram demonstrated no thrombus or patent foramen ovale, and an electrocardiogram showed normal sinus rhythm. She was discharged with subcutaneous enoxaparin as a bridge to warfarin for management of an acute renal infarction. During her hospitalization, she was treated for a migraine headache with a 1-time dose of sumatriptan.

Five days after discharge, the patient re-presented to the emergency department with nausea, vomiting, and epigastric and right-sided abdominal pain. She denied missing any doses of enoxaparin or oral warfarin, and continued to use sumatriptan for persistent headaches. Repeat urinalysis showed no white blood cells, leukocyte esterase, or blood. Repeat computed tomography showed wedge-shaped areas of hypoattenuation in both kidneys (Figure 2a), consistent with an evolving infarct of the left kidney and a new infarct of the right kidney with thrombosis of a segmental right renal artery branch (Figure 2b). Follow-up magnetic resonance angiography revealed normal aortoiliac, mesenteric, and main renal arteries, with no evidence of dissection or vasculopathy. She was placed on an intravenous 

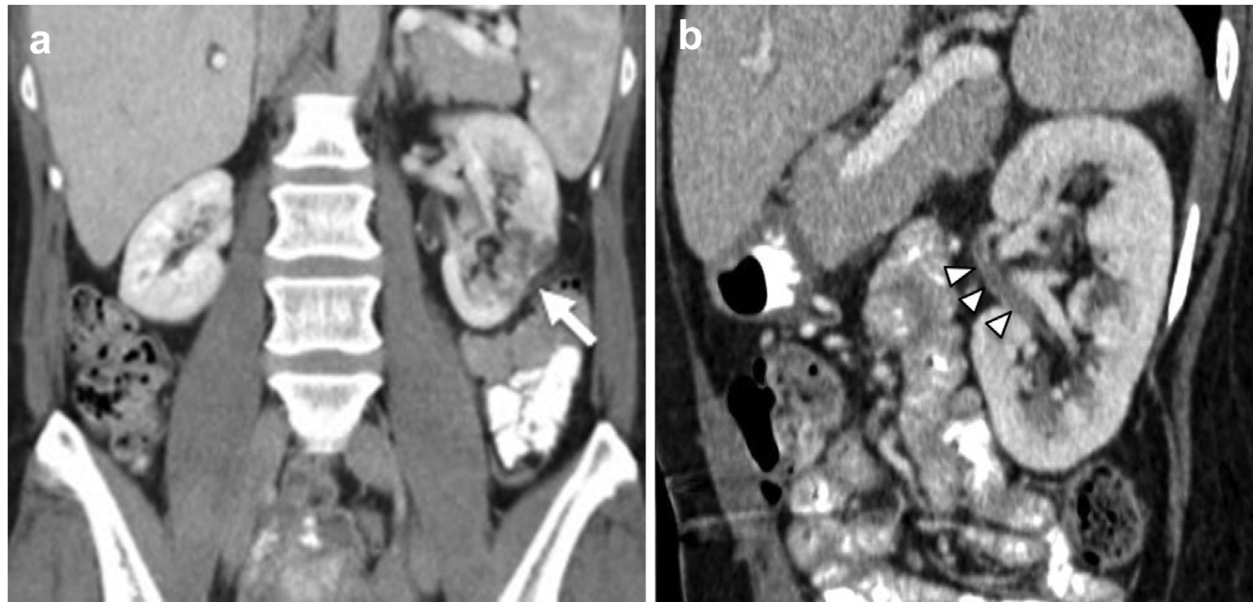

Figure 1. (a) Coronal multiplanar reformatted (MPR) image from a computed tomography examination with i.v. contrast demonstrates a peripheral, wedge-shaped area of hypoenhancement of the lower pole of the left kidney consistent with an infarct (arrow). (b) Oblique MPR image shows thrombosis of segmental artery to the infarcted region (arrowheads).

heparin drip with bridge to dabigatran. A transesophageal echocardiogram showed no cardiac thrombus or valvular vegetations. A hypercoagulable workup revealed a mildly elevated anti-cardiolipin IgM antibody (22 units; reference range, 0-12 units). Given concern for contributors to a hypercoagulable state, her intrauterine device was removed. During her hospitalization, she was treated with 2 doses of sumatriptan for migraine headaches.

Three months later, while still on dabigatran, a hypercoagulable workup was repeated. Again, there was only a mildly positive finding with anti-cardiolipin IgM antibody at 13.5 units. Anti- $\beta 2$ glycoprotein antibody measurement was within normal limits. Given the negative workup results for cardiovascular, embolic, and hypercoagulable states, her bilateral renal infarctions were attributed to sumatriptan, which was discontinued by her nephrologist. Follow-up renal function after 5 months has remained stable, and she has had no recurrence of symptoms.

\section{DISCUSSION}

Renal infarction is a rare diagnosis, resulting from compromised blood flow to the kidney, with the most common causes including thromboembolic etiologies (atrial fibrillation or infective endocarditis), renal artery dissection, or hypercoagulable states. ${ }^{7}$ Less common etiologies include fibromuscular dysplasia ${ }^{8}$ and vasospasm related to cocaine $e^{9,10}$ or ergotamine ${ }^{11}$ use. In an autopsy series of 14,411 bodies, the incidence of renal infarction was $1.4 \% .{ }^{12}$ In a retrospective study involving 18,287 patients, idiopathic or spontaneous renal infarction was noted in $0.3 \%$ of patients. ${ }^{13}$ The incidence of recurrent renal infarction is uncommon. We report a case of recurrent, bilateral renal infarcts

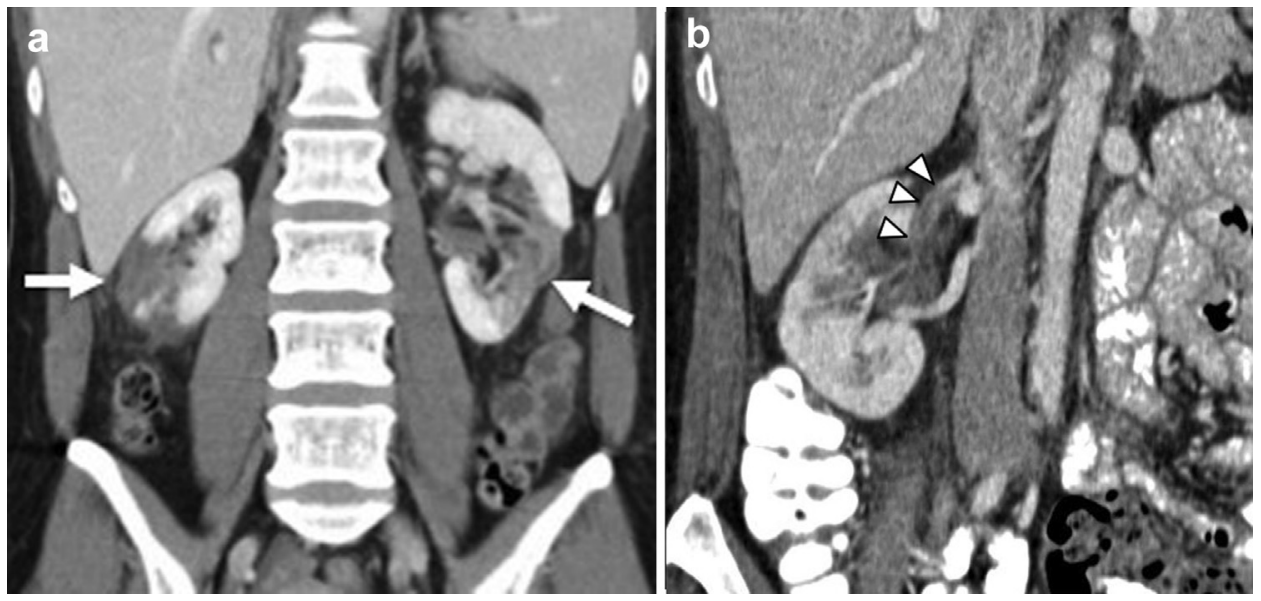

Figure 2. (a) Coronal multiplanar reformatted (MPR) image from a computed tomogram with i.v. contrast identifying areas of infarction in both kidneys (arrows). (b) Oblique MPR image shows thrombosis of segmental artery to the newly infarcted region in the right kidney (arrowheads). 
associated with sumatriptan use, while on anticoagulation, in the absence of thromboembolic or renal artery lesions.

The clinical picture of an acute renal infarction can be confounded by other more common disease states. Commonly encountered symptoms upon presentation are the acute onset of flank or abdominal pain, nausea, vomiting, and fever, which can be mistaken for acute pyelonephritis. ${ }^{14}$ Occasionally, a rise in blood pressure $^{13,14}$ can be seen due to release of renin during acute renal infarction. Typical laboratory manifestations include acute kidney injury, microscopic hematuria, and elevation of lactate dehydrogenase. ${ }^{7,15}$ The appropriate initial imaging study should be an i.v. contrast-enhanced computed tomographic scan to evaluate for wedge-shaped perfusion defects. Ultrasonography lacks the sensitivity or specificity required for the diagnosis, and the gold standard renal artery angiography is rarely performed. ${ }^{16}$ Therapy tends to be conservative, as renal tissue is not salvageable if the diagnosis is not made promptly. Antihypertensive therapy should include an angiotensin-converting enzyme inhibitor or an angiotensin II receptor blocker, given the renin-mediated hypertension. If there is concomitant atrial fibrillation, left ventricular thrombus, or a hypercoagulable state, anticoagulation should be achieved with heparin and warfarin.

Bilateral renal artery infarction is uncommon and must prompt additional evaluation for an underlying cause. The temporal relationship with sumatriptan use and renal infarction in the patient's first hospitalization, coupled with her continued use of this medication and subsequent finding of a new infarct in the contralateral kidney, suggest triptan-induced renal artery vasospasm to be the underlying culprit. Thromboembolism due to atrial fibrillation, infective endocarditis, or a hypercoagulable state were excluded with appropriate cardiac and laboratory data. Using magnetic resonance angiography, direct visualization of the renal arteries showed no anatomic abnormalities, thus making arteriopathies including renal artery dissection, fibromuscular dysplasia (FMD), and segmental arterial mediolysis (SAM) less likely. ${ }^{17,18}$ These arteriopathies, defined as idiopathic, segmental nonatheromatous diseases of unknown etiologies typically affecting small and medium-sized arteries, can be diagnosed by either computed tomographic angiography or magnetic resonance angiography. Fibromuscular dysplasia is most commonly characterized by deposition of elastic fibrils in segments of medial arterial walls, leading to arterial stenosis alternating with areas of smooth muscle loss and arterial dilation. ${ }^{19}$ Within renal arteries, this causes the characteristic "string of beads" appearance on imaging. The most common clinical finding is hypertension, which was absent in our case. Segmental arterial mediolysis was also considered, given the distinct vacuolar degeneration of smooth muscle cells within the media that differentiates this vasculopathy from FMD. ${ }^{20}$ In a systematic review of 85 cases of SAM, the renal arteries, including segmental arteries, were involved in $25 \%$ of cases. ${ }^{17}$ In a further review of the literature concerning SAM, there was a case report featuring 2 cases of SAMinduced renal infarction. ${ }^{18}$ Thrombosis of renal arteries, which was evident on magnetic resonance angiographic imaging, can occur with SAM and FMD.${ }^{19}$ However, the timing of bilateral infarctions with minimal time lapse, despite anticoagulation and with persistent use of a vasoconstrictive medication, make sumatriptanassociated renal artery vasospasm more likely.

This case identifies the first reported incidence of sumatriptan-induced renal infarction in a native kidney. The pathogenesis of this finding is likely related to renal artery vasospasm. Sumatriptan is a selective $5-\mathrm{HT}_{1 \mathrm{~B} / \mathrm{ID}}$ receptor agonist that is a potent vasoconstrictor of cerebral, coronary, and peripheral arteries. There are 5 previous reports of renal infarction due to triptan use, with only 1 case of sumatriptanassociated renal infarction in a transplanted kidney. ${ }^{21-24}$ In the case of the transplant kidney renal infarction, the presumed etiology was concomitant use of cyclosporine, a known vasoconstrictor, and sumatriptan, leading to renal infarction. Our case identified no other vasoconstrictive agents outside of the repeated sumatriptan use.

In conclusion, repeated use of sumatriptan in a healthy 45-year-old woman was associated with bilateral renal infarctions, independent of other underlying disease states including noninflammatory arteriopathies, thromboembolic disorders, or other thrombophilic or vasoconstrictive medications. Renal infarction should be considered in individuals presenting with symptoms of acute flank pain without evidence of pyelonephritis, and additional imaging evaluating renal arteries may be necessary to evaluate the cause of renal infarction. Triptans are commonly used as standard migraine therapies, given their efficacy. The potential adverse side effect of these agents of renal infarction should be recognized by prescribing physicians as an uncommon, but clinically relevant, diagnosis.

\section{DISCLOSURE}

All the authors declared no competing interests.

\section{REFERENCES}

1. Tfelt-Hansen P, De Vries P, Saxena PR. Triptans in migraine: a comparative review of pharmacology, pharmacokinetics and efficacy. Drugs. 2000;60:1259-1287. 
2. Wasson S, Jayam VK. Coronary vasospasm and myocardial infarction induced by oral sumatriptan. Clin Neuropharmacol. 2004;27:198-200.

3. Barra S, Lanero S, Madrid A, et al. Sumatriptan therapy for headache and acute myocardial infarction. Expert Opin Pharmacother. 2010;11:2727-2737.

4. Cavazos J, Caress J, Chilukuri V, et al. Sumatriptan-induced stroke in sagittal sinus thrombosis. Lancet. 1994;343: 1105-1106.

5. Knudsen JF, Friedman B, Chen M, Goldwasser JE. Ischemic colitis and sumatriptan use. Arch Intern Med. 1998;158: 1946-1948.

6. Vijayan N, Peacock JH. Spinal cord infarction during use of zolmitriptan: a case report. Headache. 2000;40:57-60.

7. Oh YK, Yang CW, Kim Y, et al. Clinical characteristics and outcomes of renal infarction. Am J Kidney Dis. 2016;67:243-250.

8. Basile C, Lisi P, Chimienti D, et al. Fibromuscular dysplasia of renal arteries presenting with bilateral renal infarction in a young man. J Nephrol. 2013;26:945-948.

9. Madhrira MM, Mohan S, Markowitz GS, et al. Acute bilateral renal infarction secondary to cocaine-induced vasospasm. Kidney Int. 2009;76:576-580.

10. Hoefsloot W, de Vries RA, Bruijnen R, Bosch FH. Renal infarction after cocaine abuse: a case report and review. Clin Nephrol. 2009;72:234-236.

11. Janssen van Doorn $K$, van der Niepen $P$, van Tussenbroeck F, Verbeelen D. Acute tubulo-interstitial nephritis and renal infarction secondary to ergotamine therapy. Nephrol Dial Transplant. 2000;15:1877-1879.

12. Hoxie HJ, Coggin CB. Renal infarction: statistical study of two hundred and five cases and detailed report of an unusual case. Arch Intern Med. 1940;65:587-594.

13. Paris $B$, Bobrie G, Rossignol P, et al. Blood pressure and renal outcomes in patients with kidney infarction and hypertension. J Hypertens. 2006;24:1649-1654.
14. Bourgault M, Grimbert $P$, Verret $C$, et al. Acute renal infarction: a case series. Clin J Am Soc Nephrol. 2013;8: 392-398.

15. Korzets Z, Plotkin E, Bernheim J, Zissin R. The clinical spectrum of acute renal infarction. Isr Med Assoc J. 2002;4: 781-784.

16. Hazanov N, Somin M, Attali M, et al. Acute renal embolism. Forty-four cases of renal infarction in patients with atrial fibrillation. Medicine (Baltimore). 2004;83:292-299.

17. Shenouda M, Riga C, Naji Y, Renton S. Segmental arterial mediolysis: a systematic review of 85 cases. Ann Vasc Surg. 2014;28:269-277.

18. Filippone EJ, Foy A, Galanis T, et al. Segmental arterial mediolysis: report of 2 cases and review of the literature. Am J Kidney Dis. 2011;58:981-987.

19. Olin J, Gornik H, Bacharach J, et al. Fibromuscular dysplasia: state of the science and critical unanswered questions: a scientific statement from the American Heart Association. Circulation. 2014;129:1048-1078.

20. Pillai AK, Iqbal SI, Liu RW, et al. Segmental arterial mediolysis. Cardiovasc Intervent Radiol. 2014;37:604-612.

21. Fulton JA, Kahn J, Nelson LS, Hoffman RS. Renal infarction during the use of rizatriptan and zolmitriptan: two case reports. Clin Toxicol. 2006;44:177-180.

22. Sharma SG, Post JB, Herlitz LC, Markowitz G. Renal cortical infarction following treatment with sumatriptan in a kidney allograft recipient. Am J Kidney Dis. 2013;61: 326-329.

23. Malacarne S, Moll S, Hadaya K, et al. Renal ischaemic injuries during the use of zolmitriptan for treatment of migraines in a transplanted patient under tacrolimus therapy. Nephrol Dial Transplant. 2007;22:3341-3343.

24. Akshintala D, Bansal SK, Emani VK, Yadav M. Renal infarct: a rare disease due to a rare etiology. J Community Hosp Intern Med Perspect. 2015;5:1-2. 